
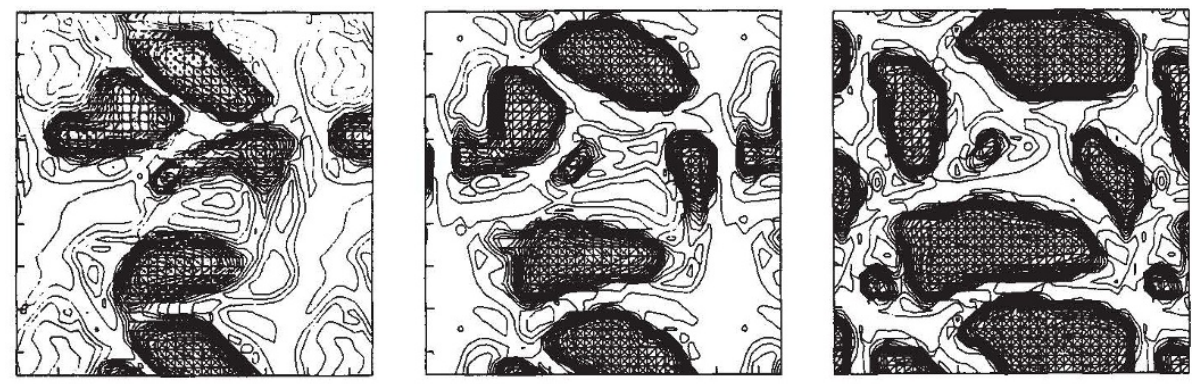

FIG. 2 Evolution, at 108-s intervals, of granules on a stellar surface in Dravins and Nordlund's model. (Sequence runs left to right, top then bottom.) Granules are represented here as hatched (darker) areas. (Taken from ref. 1.)
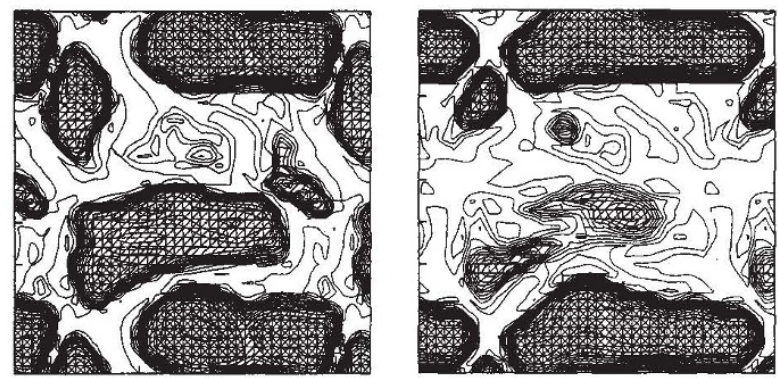

stop well below the visible surface.

Looked at from a long way off, the stellar surface presents radiation from a whole spread of velocities, temperatures and pressures. This is in very marked contrast to the simple, two-dimensionally homogeneous, stellar-atmosphere models that are typically used in the analysis of stellar spectra. It would be impossible to analyse every stellar spectrum using the complexities of these new simulations, but the calculations are very important in showing up the limitations and strengths of the simplified models. An important result is that the spectral absorption lines show broadening and profiles that are quite typical of observations. For nearly 50 years, controversy has raged about the reality or otherwise of hypothetical turbulent velocity fields in stellar atmospheres which are invoked ${ }^{3}$ to explain the observed shapes and widths of spectral lines. These turbulent velocity fields, particularly the so-called 'microturbulence', were strongly attacked as unphysical in the pages of Nature in the early 1970 s. But, lacking anything better, stellar analysts have continued to use them. What the new calculations show is that the combination of temperature and pressure inhomogeneities, and convective mass motions, is sufficient to provide the line broadening (in most cases) without having to introduce further turbulent velocity fields.

It remains to be proved that reasonable abundances of chemical elements can still be deduced by using the convenience of introducing a microturbulence broadening parameter if required for strong lines, and that the weaker lines (whose strengths are less affected by velocity fields) can be analysed with a mean vertical atmospheric structure of temperature and pressure. At least there is now a reasonable physical model of which such questions can be asked. The success that
Dravins and Nordlund have had in their detailed modelling of lines from a few stars using abundances and mean atmospheric physical conditions deduced from classical analyses argues that classical analysts may (when not observing) sleep a little easier at nights.

Some observational confirmation of the general features of the stellar granulation models comes from careful study of the asymmetry of spectral line profiles in particular the shape of the locus formed by the mid points between the positions of equal intensity down either side of an absorption-line profile. These line bisectors have shapes that are influenced by the horizontal spatial inhomogeneity of the stellar surface. Dravins $^{5}$ has already been able to make some estimate of the relative areas of surface covered by rising granules in stars of different temperature.

The current computational models cannot be regarded as complete because, as well as not including magnetic fields, the calculations assume that the fluids involved are incompressible. This eliminates any effects due to wave motions. As the authors admit, wave motions may have some part to play both in energy transport and in line broadening. But to make really significant further progress it is estimated that a further two orders of magnitude in computing power is needed. It would be a rash man who claimed this will not have happened before another ten years have passed.

M. G. Edmunds is in the Department of Physics, University of Wales, PO Box 913, Cardiff CF1 3TH, UK

1. Nordlund, $\AA$. \& Dravins, D. Astr. Astrophys. 288, 155183; 184-202; 203-217 (1990).

Schwarzschild, M. Astrophys. J. 195, 137-144 (1975)

Struve, D. \& Elvey, C.T. Astrophys, J. 79, 409 (1934).

4. Worrall, G. \& Wilson, A.M. Nature 238, 15-18 (1972)

. Dravins, D. Astr. Astrophys. 228, 218-230 (1990).

\section{Nervous tapping}

ANIMAL nerve fibres are extremely well insulated from each other, and impulses travelling along them create almost no external field. This minimizes their mutual interference and cross-talk, but hampers those neurologists who want to eavesdrop on their traffic. Now Daedalus is coming to the rescue. He points out that the ion flow accompanying a nerve impulse alters the volume of the nerve slightly, and therefore launches an ultrasonic wave into the surrounding tissue. A modern sensitive piezoelectric transducer should be able to detect this wave at the skin surface. Indeed, a pattern of such transducers, registering the time of arrival of the ultrasonic wave at various points on the skin, could even locate the origin and direction of the wave.

So Daedalus is inventing neuroseismology. DREADCO's engineers are building piezoelectric armbands, anklets, necklaces, and so on, designed to read the nerve traffic in the arms, legs and necks of volunteers. The ultrasonic disturbance accompanying a moving nerve impulse will race out ahead of it and behind it, producing at the armband a complex 'signature' encoding the position, velocity and direction of the impulse. Modern nanosecondresolution electronics and high-speed data processing will be stretched to the limit to disentangle the superimposed signatures of many close-packed nerves all firing rapidly, and to identify them by location and transmission velocity; but the thing seems feasible. Once the pilot rig is working, the DREADCO team will ask their volunteers to bend each finger in turn, for example, or prick their hands in various places, so as to build up a 'map' of which nerves do what in the arm.

When perfected, the neuroseismograph will be a wonderful diagnostic tool. All those mysterious aches and pains that are so hard to pin down will at last be located and scrutinized. The nerve traffic sustaining muscular tics and spasms, phantom sensations and the like, will also be immediately apparent. A sequence of neuroseismograms made over time would give a marvellously detailed insight into the progress of treatment of such disabilities.

But Daedalus reckons his neuroseismograph will be most useful as a biofeedback monitor. All sorts of skills, like those of the golfer, musician, and typist, depend on establishing proper reflexes in the arms. A trainee wired to a neuroseismograph could immediately compare his nerve recordings with those of an expert and see where he was going wrong. He could polish his skills until the right muscles were receiving the most effective pattern of reflex commands for the job. Even the trickiest skill of all, complete muscular relaxation, could at last be easily assessed and improved.

David Jones 